\title{
Flaps with Skin Islands for Managing Skin Defects after Breast Conserving Surgeries
}

\author{
Ahmed G. Osman, MD; ${ }^{1}$ Ahmed Maher Moslem, MD; ${ }^{2}$ Ehab H AbdEI-wahab, MD; ${ }^{1}$ Mohammad \\ Ahmad Abd-erRazik, MD ${ }^{1}$ \\ ${ }^{1}$ Department of General Surgery, Faculty of Medicine, Ain Shams University, Egypt \\ ${ }^{2}$ Department of General Surgery, Faculty of Medicine, New Giza University, Egypt
}

Background: The breast cancer is the most common malignancy in Egyptian women. The management of breast cancer evolves with time. The breast conserving Surgery (BCS) thereby, is now the gold standard when managing cases with early breast cancer, it is composed of BCS and adjuvant radio therapy. However, managing cases with locally advanced breast cancer is more challenging.

Patients and methods: This was a concurrent cohort study, conducted in Ain Shams University Hospitals, between June 2017 to June 2021. Patients were with age ranging from 20 and 60 years. Patients were suffering of malignant breast masses, which is either T1-3, near to the skin, with no skin involvement, or a previously T4b mass and the patient received neo-adjuvant therapy with complete clinical resolution of the skin; T3 masses should have received neoadjuvant therapy and had a good response, and patients were accepting the procedure. Patients with the following criteria were excluded: presence of residual skin involvement after the neoadjuvant therapy or inflammatory breast cancer, multicentric breast cancer, those with history of BCS on the same side, patients with contraindications or refusing postoperative radio therapy or heavy smokers. Patients $(n=31)$ underwent dermoglandular or dermo-muscular flaps with skin islands after BCS and limited skin excision.

Results: The mean age was $43.8 \pm 6.4$, the mean BMI was $35 \pm 5.6 \mathrm{~kg} / \mathrm{m} 2$. The most prevalent cup size was D $(35.5 \%)$. The breast masses had a mean size of $3.5 \pm 1.1 \mathrm{~cm}$. Early post operative complications were reported in $16.1 \%(n=5)$ of the patients. The most recorded complication was wound dehiscence $12.9 \%$ of the patients, a single patient suffered of wound hematoma. No recoded cases of wound infection or flap necrosis among the study group. Seroma was recorded in $9(29 \%)$ patients. No local recurrences were reported. The esthetic results were Good in $81 \%$, Fair in $13 \%$ and Poor in $6 \%$.

Conclusion: The DGF and DMF with skin islands are feasible, safe, with good esthetic results for the repair of the skin defects resulting from skin excision during the breast conserving surgeries.

Key words: Breast cancer, skin affection, T4b, dermo-glandular flap, dermo-muscular flap.

\section{Introduction}

In the time of writing this manuscript, there is more than 7.8 million alive females diagnosed with breast cancer in the past five years. More than 2.3 million females were diagnosed with breast cancer in 2020 globally, resulting in more than 685 thousand deaths. Breast Cancer is the most prevalent cancer worldwide, it occurs at any age after puberty, whoever the rate increases in later life, it occurs in every country of the world.

The breast cancer is the most common malignancy in Egyptian women, with an incidence rate of $18 \%$ and a mortality rate of $10.8 \%$. Based on the current trend of the disease, the forecasted number of cases of breast cancer patients in Egypt in 2040 is more than 40 thousand cases. ${ }^{2}$

The management of breast cancer evolves with time. Since Halsted described his technique, ${ }^{3}$ the trend was to migrate from the "maximum tolerable treatment" to the "minimum effective treatment". ${ }^{4}$ That is why techniques like the breast conserving surgery (BCS). ${ }^{5}$ and the oncoplastic breast surgery
(OBS). ${ }^{6}$ are popular now, if they can achieve oncological safety for the patient. Moreover a technique like the extreme oncoplasty (EO). ${ }^{7}$ was proposed to extend the indications of the BCS. The evolution was also in the diagnostic methods and the chemo and radio therapy protocols.

The breast conserving thereby (BCT), is now the gold standard when managing cases with early breast cancer, it is composed of BCS and adjuvant radio therapy (RT). However, managing cases with locally advanced breast cancer is more challenging. The locally advanced breast cancer is defined as tumors larger than $5 \mathrm{~cm}$, i.e., T3 tumors, or tumors with skin or chest wall involvement, i.e., T4 tumors. ${ }^{8}$

Classically the T4b tumor is defined as a tumor, of any size, with a "limited" area of edema (Including peau d'orange) or ulceration of the breast skin, or gross satellite skin nodules (not only seen by microscope) limited to the same breast. But other skin changes, like the skin dimpling, the retraction of the nipples, or any other skin changes (Except T4b and inflammatory breast cancer [T4d]) can occur with $\mathrm{T} 1, \mathrm{~T} 2$, or $\mathrm{T} 3$ tumors without changing 
the classification. ${ }^{9}$

In this study, we performed 2 techniques to substitute skin defects after performing BCS / OBS in certain population of breast cancer patients, in which a limited skin resection was necessary.

\section{Patients and methods}

The study was a concurrent cohort study, conducted on female patients with breast cancer, presented to breast clinics in Ain-Shams University Hospitals. The study period was between June 2017 to June 2021.

Recruited patients were a very specific population; they were females with age ranging from 20 and 60 years old. Patients were suffering of malignant breast masses, which is either T1-3, near to the skin, with no skin involvement, or a previously T4b mass and the patient received neo-adjuvant therapy with complete clinical resolution of the skin; T3 masses should have received neoadjuvant therapy and had a good response, and patients were accepting the procedure.

Patients with the following criteria were excluded: presence of residual skin involvement after the neoadjuvant therapy or inflammatory breast cancer, multicentric breast cancer, those with past history of BCS on the same side, patients with contraindications or refusing postoperative radio therapy, heavy smokers, patients suffering of multiple comorbidities, metastatic disease or those who were not fit for anesthesia.

The study was approved by the Research Ethics Committee (REC) of the General Surgery Department, Ain-Shams University (IRB 00006379). All patients signed out an informed consent after explanation of the procedure and the alternatives.

\section{Procedures}

All potentially candidate patients were subjected to full history taking and full general and local examination. Followed by sono-mammography and if indicated, contrast enhanced mammography or magnetic resonant imaging with contrast. Core needle biopsy was done for all patients, to identify the tumor type, grade, and the molecular markers. The multidisciplinary team (MDT) of the Breast Surgery Department, Ain-Shams University reviewed all patients' data, and the discissions were tailored for each patient. Those who fulfilled the inclusion / exclusion criteria were prepared for surgery as per our hospital protocol.

Preoperative skin marking was done in the day of surgery, (Fig. 1). A wide local excision of the mass, a circumferential margin and the adjacent skin was done. After labeling it, the specimen was sent for frozen section, (Fig. 2). The tumor bed was marked by titanium clips. Axillary dissection or sentinel lymph node excision was done according to the dissension taken by the MDT. The flap design was decided according to the breast size, the place and size of the tumor as well as the diameter of the excised skin. All the flaps had a skin island to replace the skin defect.

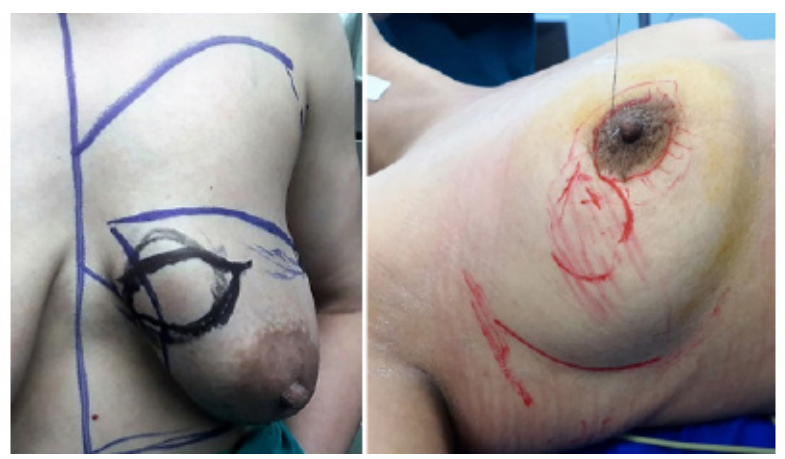

Fig 1: Preoperative marking.

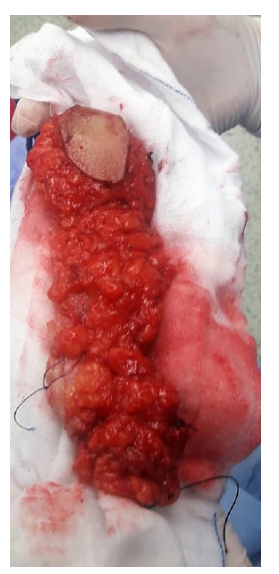

Fig 2: Marking the specimen for frozen section.

In small breasts i.e., smaller than size D, a dermomuscular flap was performed, using the latissimus dorsi (LD) muscle with a skin island. Via an elliptical incision in the back, (Fig. 3), the LD muscle was harvested, depending on the thoracodorsal bundle. A tunnel was created to conduct the flap to the area of the tissue defect.

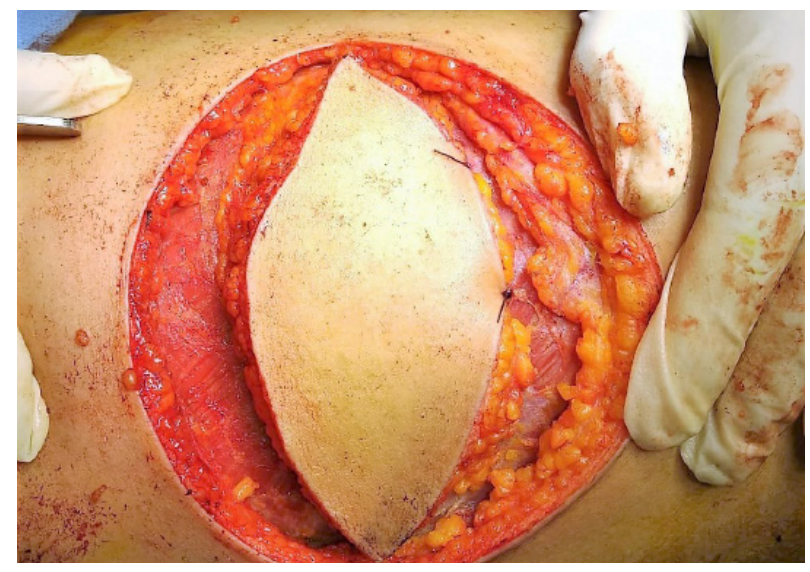

Fig 3: Elliptical incision over the latissimus dorsi muscle. 
While in larger breasts i.e., size D and above, a dermo-glandular flap with skin island was performed, and the technique is tailored according to the site of the skin defect area. If the defect was at the nipple-areola complex (NAC) area, the skin island was dissected depending on an inferior pedicle, (Fig. 4). However, if the skin defect was away from the NAC, breast reduction was done on a superomedial pedicle flap, then another inferior pedicle flap with skin island was dissected and conducted to the area of the tissue and skin defects, (Fig. 5).

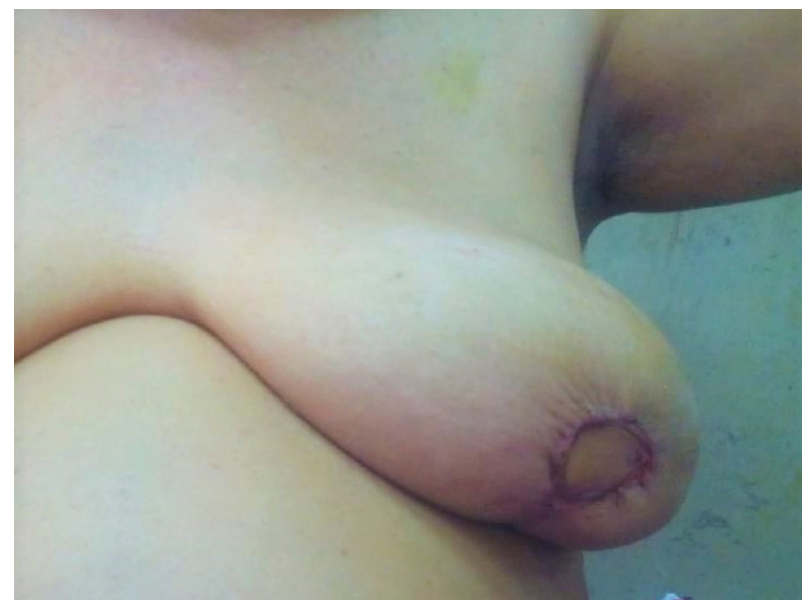

Fig 4: Skin replacing the NAC.

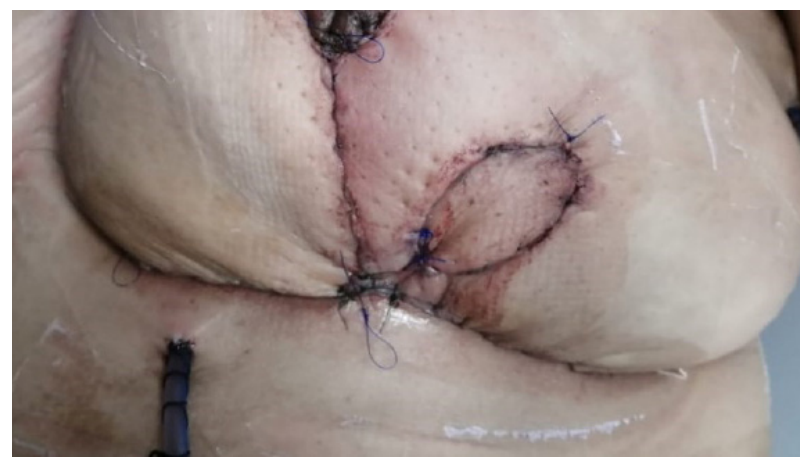

Fig 5: A DGF with reduction mammoplasty.

Being in place, the flaps were secured by absorbable sutures. Suction drains were inserted in all patients, and the skin was closed

\section{Follow-up}

The overall follow-up period ranged from 3-4 years. After surgery there were a weekly follow-up visit for a month, then a monthly visit for 6 months, then a yearly visit. The esthetic results were assessed by the MDT along the follow-up visits, (Fig. 6). Patients were monitored and assessed to exclude the presence of complications in the early follow up visits and were assessed for the presence of local or systemic recurrence after wards.
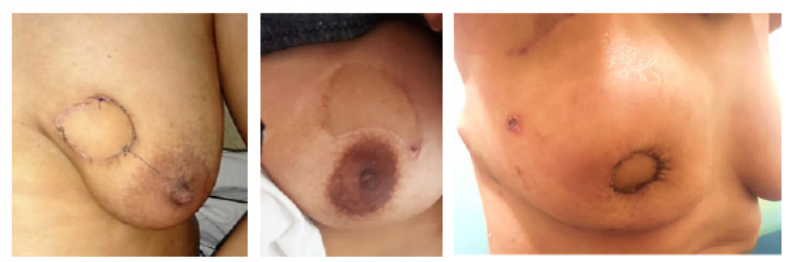

Fig 6: Postoperative results.

\section{Statistical Analysis}

The collected data had been coded and tabulated by using Excel 365, Microsoft Corporation, USA. The statistical analysis was done using SOFA statistics (Version 1.5.4), Paton-Simpson \& Associates Ltd., Auckland, New Zealand. The continuous data were expressed as mean/median $\pm S D$, while the categorical data were presented as frequencies and percentages.

\section{Outcome}

The study aimed to identify the feasibility, safety, and esthetic outcome of the DGF and the DMF techniques for managing patients with limited skin defects during BCS / OBS. And to record some operative data and complications resulted from using those techniques.

\section{Results}

This study was conducted on female patients suffering of breast cancer. The age of the recruited patients ranged from 33-59 years; the body mass index (BMI) ranged from $25-45 \mathrm{~kg} / \mathrm{m}^{2}$. The most prevalent cup size was D (35.5\%). Twenty-nine percent of the patients were menopausal and $16.1 \%$ of them were smokers. Patients' characteristics and comorbidities were presented in (Table 1).

The recruited patients had breast masses ranging in size, measured radiologically, between 1.5 and $5.6 \mathrm{~cm}$, with mean size of $3.5 \pm 1.1 \mathrm{~cm}$. All the patients $(n=31)$ were found to have invasive ductal carcinoma, diagnosed by the core needle biopsy. Nearly $80 \%(n=25)$ of the patients received neoadjuvant chemotherapy before joining the study. Most of the patients underwent DMF technique, the operative data were represented in (Table 2).

Early post operative complications were reported in $16.1 \%(n=5)$ of the patients. The most recorded complication was wound dehiscence, which was recorded in $4(12.9 \%)$ of the patients, a single patient $(3.2 \%)$ suffered of wound hematoma. No recoded cases of wound infection or flap necrosis 
among the study group. Seroma was recorded in 9 (29\%) patients. Along the whole follow-up period no local recurrences or a metastatic disease were detected among the patients. The esthetic results of the procedures, which was evaluated by the MDT, were represented in (Fig. 7).

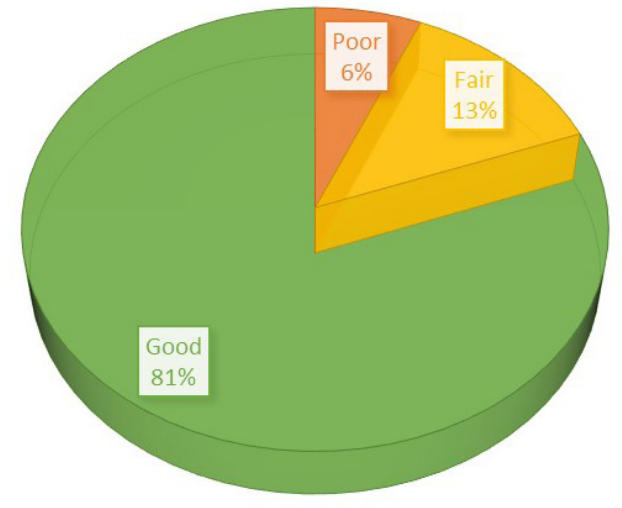

Fig 7: The esthetic results according to the MDT opinion.

\begin{tabular}{lcc}
\hline & & All patients $(\mathbf{n}=\mathbf{3 1})$ \\
\hline Age & & $43.8 \pm 6.4$ \\
Cup size & & $35 \pm 5.6$ \\
& B & $6(19.4 \%)$ \\
& C & $5(16.1 \%)$ \\
Menopause & D & $11(35.5 \%)$ \\
& $>$ D & $9(29 \%)$ \\
DM & Yes & $9(29 \%)$ \\
& No & $22(71 \%)$ \\
HTN & Yes & $4(12.9 \%)$ \\
& No & $27(87.1 \%)$ \\
Smoking & Yes & $6(19.3 \%)$ \\
& No & $25(80.7 \%)$ \\
\hline
\end{tabular}

BMI: Body mass index, DM: Diabetes mellites, HTN: Hypertension.

Table 2: Operative data

\begin{tabular}{llc}
\hline & & All patients $(\mathbf{n}=\mathbf{3 1})$ \\
\hline Procedure & DGF & $20(64.5 \%)$ \\
& DMF & $11(35.4 \%)$ \\
Area of excised skin & $\mathrm{cm}$ & $4.2 \pm 0.8$ \\
Blood loss & $\mathrm{CC}$ & $76.2 \pm 27.7$ \\
Operative time & minutes & $181.2 \pm 26.3$ \\
\hline
\end{tabular}

DGF: Dermo glandular flap, DMF: Dermo muscular flap. 


\section{Discussion}

In a study involved 7316 cancer patients, 367 $(5.0 \%)$ cases of them were reported to have skin involvement as a presenting sign of their internal malignancy. The breast cancer was the most common cause of the direct invasion and the local metastases to the skin. ${ }^{10}$ The skin involvement was, always, an indication of a mastectomy (MRM), either before or after neoadjuvant therapy, to achieve good local controller.

A study involved 453 non-metastatic, noninflammatory, breast cancer patients, 81 patients of them were T4b. The patients were distributed into 3 groups according to the size of the tumor only, neglecting the skin involvement as a factor. Then the patients were compared to a control group of 372 patients, none of them had skin involvement. That study concluded that there were no significant differences in the disease-specific survival between both groups. The multivariate analysis showed that the skin involvement wasn't a major predictor of the disease-specific survival. They also concluded that the maximum T classification, or Stage III, is not the proper categorization for a considerable sum of patients having T4b masses, according to the current classifications, due to the heterogenicity of this category. ${ }^{11}$

That's why studies had examined the possibility and the consequences of performing BCS for those patients. A study recruited 98 patients with clinical T4 M0 patients, $32 \%$ of them had T4b masses, had concluded that the careful evaluation of the pathology and neoadjuvant therapy response may identify patients with clinical T4 candidate for breast conservation. ${ }^{12}$ According to the NCCN guidelines, version 1.2021, the BCS may be performed in a selected group of patients with $\mathrm{T} 4$, non-inflammatory patients, if there is complete resolution of the skin involvement after the neoadjuvant therapy. ${ }^{13}$

On the other hand, some skin defects may inevitably happen when trying to achieve a tumor free concentric safety margin, even if the skin was not involved. In both scenarios (the skin involvement after resolution or very near free skin) the anticipated defect pushes the patient towards a MRM. That decision may potentially alter the patient's perception of her sexual and physical wholeness. ${ }^{14}$

Skin defects resulted from the tumors excisions in this study ranged from 3 to $6 \mathrm{~cm}$ in diameter, with average of $4.2 \mathrm{~cm}$. Primary closure of those defects will cause distortion of the breast contour and deviation of the NAC, as it will augment the distortion caused by the excision of a volume of breast tissue, and the smaller the breast the larger the distortion.
We used dermo-glandular flaps and dermo-muscular flaps with skin islands to fill the skin defects resulted from skin excision during the BCS. The choice of the procedure was tailored according to the size of the breast and the diameter of the excised skin. The latissimus dorsi flaps replaces breast volume defect resulted from wide local excision in a relatively small breast, at the same time it provides the blood supply to the skin island. While in larger breast sizes, a dermo-glandular flap will provide the blood supply to the skin island, the procedure may be even combined with a reduction mammoplasty. Some patients may require a contra lateral breast symmetrization after those procedures. We excluded heavy smokers from this study as they may face higher rates of flap loss, ${ }^{15}$ also smokers were advised to stop smoking immediately till the time of surgery and at least a month after the procedure.

Postoperative complications were minimal, except for seroma formation. Some studies considered transient seromas, that doesn't need intervention, as a sequalae rather than a complication. ${ }^{16}$ Seromas may occur in the breast, axilla and / or at the LD doner site in patients underwent latissimus dorsi flaps.

Many studies had reported that the five-years local recurrence-free survival rates after neoadjuvant chemotherapy for advanced breast cancer is little pit better in patients undergoing mastectomy if compared to BCS, but this difference is not statistically significant difference i.e., they are comparable. ${ }^{17}$ Along the follow up period which extended up to 3 years, none of the patients showed local recurrence.

This study approached a specific population, tried to tailor a safe alternative with better esthetic outcome. Acceptable results were achieved in the vast majority of the patients and putting in consideration that the alternative would be a mastectomy, those results are more appreciated. The personalization of the BCS would consider the medical aspects of the process and also should consider the specific preferences and needs of the patients, as humans. ${ }^{18}$

\section{Conclusion}

The dermo-glandular flaps and dermo-muscular flaps with skin islands are feasible, safe tools, with good esthetic results for the repair of the skin defects resulted from skin excision during the breast conserving surgeries. Controlled studies with larger sample number and longer follow-up periods are needed.

\section{References}

1. DeSantis CE, Bray F, Ferlay J, et al: International variation in female breast cancer incidence and mortality rates. Cancer Epidemiol 
Biomarkers Prev. 2015; 24(10): 1495-1506. doi:10.1158/1055-9965.EPI-15-0535

2. National Plans | ICCP Portal: WHO - Cancer country profiles Egypt 2020. Accessed October 19, 2021. https://www.iccp-portal.org/map.

3. Halsted WS: A Clinical and histological study of certain adenocarcinomata of the breast: And a brief consideration of the supraclavicular operation and of the results of operations for cancer of the breast from 1889 to 1898 at the Johns Hopkins Hospital. Ann Surg. 1898; 28(5): 557.

4. Veronesi U, Stafyla $V$, Luini $A$, et al: Breast cancer: from "maximum tolerable" to "minimum effective" treatment. Front Oncol. 2012; 0: 125. doi:10.3389/FONC.2012.00125.

5. Miller $K D$, Nogueira $L$, Mariotto $A B$, et al: Cancer treatment and survivorship statistics, 2019. CA Cancer J Clin. 2019; 69(5): 363-385. doi:10.3322/caac.21565.

6. Koppiker $\mathrm{CB}$, Chintamani, Dixit S: Oncoplastic breast surgery in India: Thinking globally, acting locally. Indian $\boldsymbol{J}$ Surg. 2019; 81(2):103110. doi:10.1007/s12262-019-01890-8.

7. Franceschini G, Di Leone A, Masetti R: Comment on "extreme oncoplastic surgery for multifocal/multicentric and locally advanced breast cancer." Int J Breast Cancer. 2019; 2019. doi:10.1155/2019/4693794.

8. Hunt KK, Ames FC, Singletary SE, et al: Locally advanced noninflammatory breast cancer. Surg Clin North Am. 1996; 76(2): 393-410. doi:10.1016/s0039-6109(05)70446-1.

9. Connolly JL: Changes and problematic areas in interpretation of the AJCC cancer staging manual, $6^{\text {th }}$ Edition, for Breast Cancer. Arch Pathol Lab Med. 2006; 130(3): 287-291. doi:10.5858/2006-130-287-CAPAII.
10. Lookingbill DP, Spangler N, Sexton FM: Skin involvement as the presenting sign of internal carcinoma: A retrospective study of 7316 cancer patients. J Am Acad Dermatol. 1990; 22(1): 1926. doi:10.1016/0190-9622(90)70002-Y.

11. GüthU, WightE, Schötzau A, etal: Anewapproach in breast cancer with non-inflammatory skin involvement. Acta Oncol (Madr). 2006; 45(5): 576-583. doi:10.1080/02841860600602953.

12. Murphy BL, Hoskin $\mathrm{TL}$, Boughey $\mathrm{JC}$, et al: Contemporary operative management of T4 breast cancer. Surgery. 2016; 160(4): 10591069. doi:10.1016/j.surg.2016.06.030.

13. National Comprehensive Cancer Network. Breast cancer (Version 1.2021).; 2021.

14. Pillai-Friedman S, Ashline JL: Women, breast cancer survivorship, sexual losses, and disenfranchised grief - a treatment model for clinicians. Sex Relatsh Ther. 2014; 29(4): 436453. doi:10.1080/14681994.2014.934340.

15. Hwang K, Son JS, Ryu WK: Smoking and flap survival. Plast Surg. 2018; 26(4): 285. doi:10.1177/2292550317749509.

16. Dey A: Should seroma be considered a complication? Hernia 2021. 2021; 1: 1-2. doi:10.1007/S10029-021-02385-W.

17. Zhou $X, L i$ Y: Local recurrence after breastconserving surgery and mastectomy following neoadjuvant chemotherapy for locally advanced breast cancer - a meta-analysis. Breast Care. 2016; 11(5): 345. doi:10.1159/000450626.

18. Catsman CJLM, Beek MA, Voogd AC, et al: The COSMAM TRIAL a prospective cohort study of quality of life and cosmetic outcome in patients undergoing breast conserving surgery. BMC Cancer. 2018; 18(1): 1-7. doi:10.1186/S12885018-4368-8. 